\title{
Acquiring After-Sales Knowledge from Human Motions
}

\author{
Satoshi HORI ${ }^{1}$, Kota HIROSE ${ }^{1}$, Hirokazu TAKI ${ }^{2}$, \\ ${ }^{1}$ Monotsukuri Institute of Technologists \\ 333 Maeya, Gyoda 361-0038 JAPAN \\ hori@iot.ac.jp \\ http://www.iot.ac.jp/manu/HORI.html \\ 2 Wakayama University \\ 930 Sakaedani, Wakayama 640-8510 JAPAN
}

\begin{abstract}
Human motion is a key property to understand human's knowledge and intention. A service technician moves to achieve his duty: facility maintenance. The series of his motions is the output of his observation, diagnosis, and repair. Therefore the motions are recognized as a knowledge source. From the viewpoint of after-sales productivity, improving service technicians' skill is a vital key to quick and efficient service. However recording service cases costs high and is a bottleneck of knowledge and experience sharing systems. We propose a framework that measures human motion behaviors and elicits knowledge from the motions to overcome the bottleneck. This article describes the knowledge acquisition framework and how IC accelerometers can measure human motions. Empirical results that prove its effectiveness are also shown.
\end{abstract}

\section{Introduction}

After-sales service is one of promising business sectors whose market and profit are growing. Improving service technicians' skill is a vital key to quick and efficient service and service experience sharing is proven to improve service productivity. However recording service cases costs high and is a bottleneck of knowledge and experience sharing systems. This article describes the knowledge acquisition framework and how IC accelerometers can measure human motions. Empirical results that prove its effectiveness are also shown.

The background and objectives of this research is explained in the following section. Section 2 describes the outline of the proposed method and reviews briefly previous works. Section 3 explains the algorithm of measuring human motion with IC accelerometers as a part of knowledge acquisition. 


\subsection{Background and Objectives}

At home and business our society operates many facilities such as home appliances, air-conditioning systems, and power electric equipments. After-sales service is a business exercise such that a dispatched service technician maintains a facility work properly. He or she trouble-shoots and repairs the facility when it is malfunctioning. The after-sales service is now one of the most profitable and fast-growing business sectors. As an example, General Electric launched a jet engine service business in which GE monitors operating jet engines. This service provides airliners with more efficient jet engine operation. Therefore this service reportedly results in high profit to GE.

Advance of information technology enables us easily to monitor and control equipment located in distance. Many expert systems [1, 4] were developed to infer malfunction. As an intelligent system, CBR, Case-Based Reasoning [2], has been adopted in this field because a previous work [3] also shows that experiment sharing is a good way to support service technicians in troubleshooting. However the bottleneck of these intelligent systems are recording electronically repair cases with lower cost. Therefore a method of recording what a service technician executes at after-service practice has been keenly demanded. A conventional way of recording the cases is letting service technicians to write service reports. Writing service report is very timeconsuming task for service technicians, hence this method costs high.

Therefore the research objective is to develop a system that can acquire service knowledge from the maintenance experience without bearing a burden on human technicians to record the experience. Our method measures service technician's motions by electronics sensors, and then infers his/her task from the recorded motions.

\subsection{Application Scenario}

This section illustrates how our KA, Knowledge Acquisition, from motion can work in order to demonstrate its usefulness in a real-world situation. Let's consider Start-up task of an industrial robot shown in figure 1 as an example.

Assuming service technician A is usually in charge with this start-up task. He encountered "sensor alarm " during the start-up and discovered a closed air-valve is a cause of the alarm. It took one hour for him to troubleshoot.

His motion behaviors were measured. His motion and ordinary start-up steps are compared and the mismatch is detected as figure 2 shows. Finally a new branch of a diagnostic tree is added after the service technician added short comments.

Service technician B happened to perform the start-up maintenance instead of technician A. He accidentally encountered the sensor alarm that he has no knowledge of. However technician B retrieved the case that technician A experienced before, and easily resolved the alarm by opening the air-valve. 


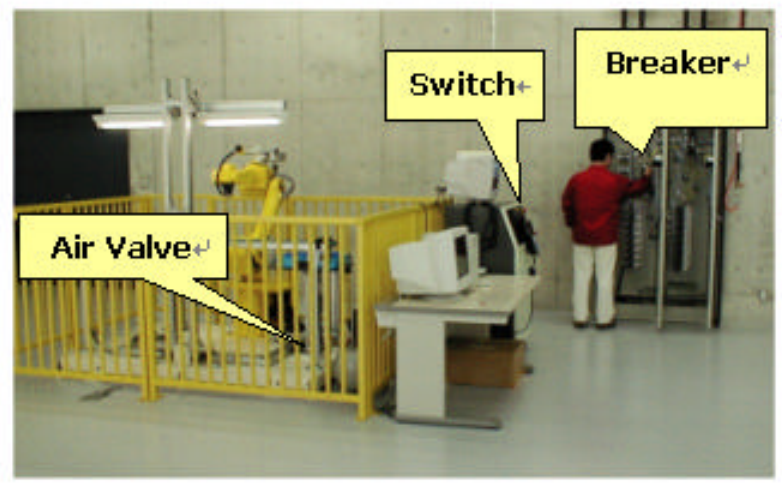

Fig. 1. Start-up of Industrial Robot. The usual start-up procedure is defined as below: STEP-1. Turn on a breaker. STEP-2. Turn on a power breaker of control box. STEP-3. Turn on a power switch of control box. STEP-4. Push start button when system becomes ready.
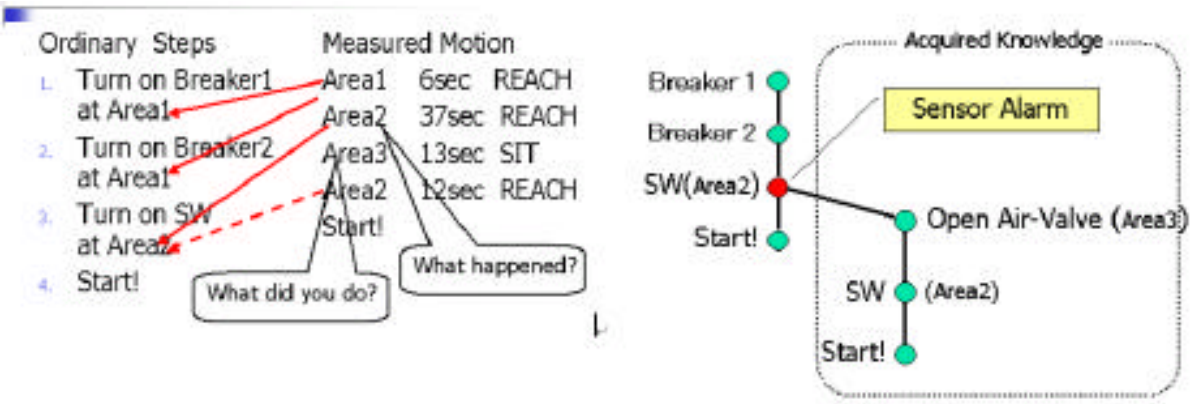

Fig. 2. Acquiring a new branch. Motions were measured. The motions and ordinary start-up steps are compared and the mismatch is detected. The mismatched data are acquired as a new experience. Finally a new branch of a diagnostic tree is added after the service technician added short comments.

\section{Knowledge Acquisition from Motion}

In this section, our method is outlined, and then previous works is briefly reviewed.

\subsection{Outline of Proposed Method}

The proposed method stores service records as Figure 3 shows. As a result, we can semi-automatically build a casebase of service experience. 
Step- 1 Measure motion accelerations by IC sensors attached to legs and arms of a service technician. Time and location are also recorded.

Step- 2 Decide what basic motion he moved, from the data recorded in step-1.

Step- 3 Infer service task from the series of basic motions elicited at step-2.

Step- 4 Service technician confirms the service task generated in step-3 and adds comment if necessary after completing his maintenance task.

Step- 5 Store the confirmed service task in a casebase as a diagnostic tree form.

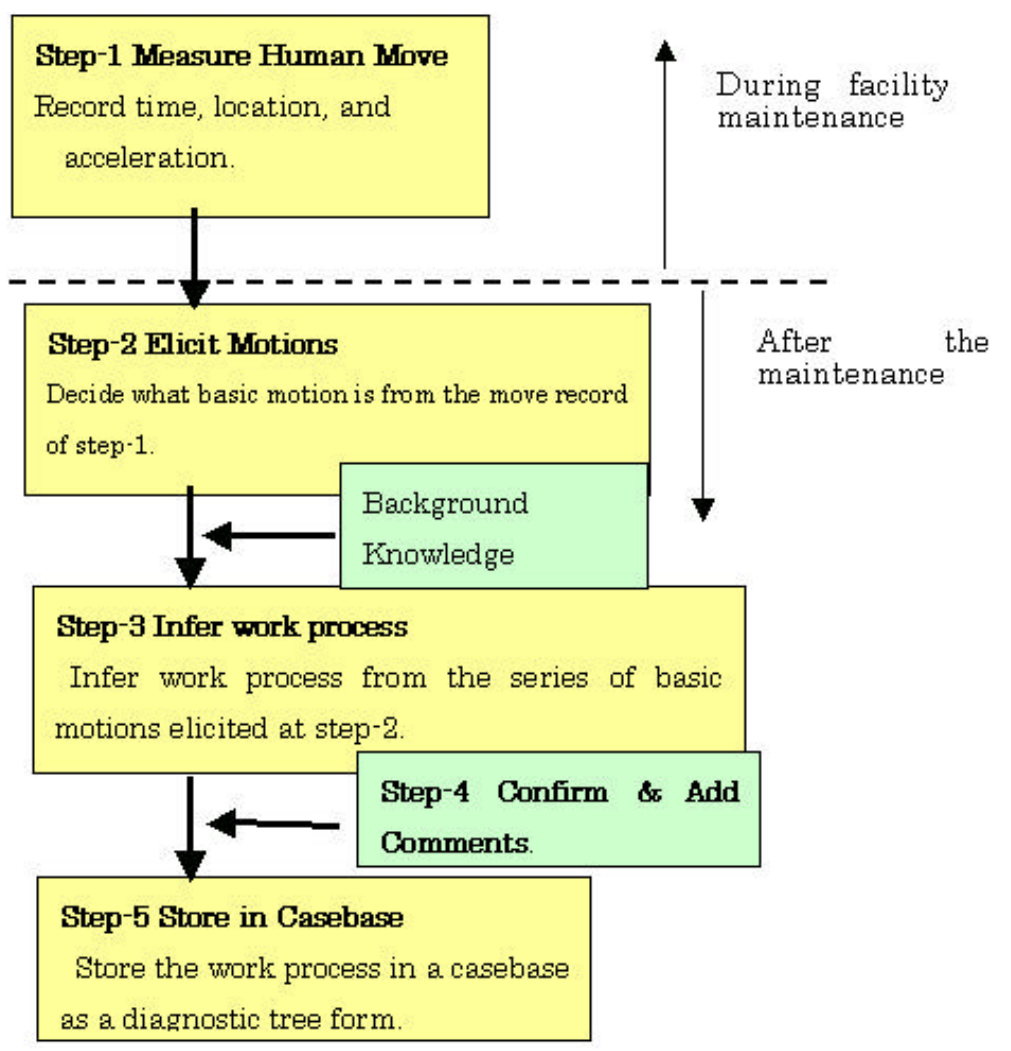

Fig. 3. Knowledge Acquisition from Motion

\subsection{Previous Works}

This section reviews briefly previous works related to after-service knowledge acquisition. The knowledge acquisition has been recognized as a bottleneck of conventional expert systems. A complete, accurate and updated knowledge base is essential for an expert system to conduct accurate diagnosis. However making a good knowledge base is a time consuming and difficult process. Model-based reasoning [5], rule induction [6] and case-based reasoning [2] have been proposed to overcome this bottleneck. 
The CBR has been employed for several diagnostic systems [3] because previous troubleshooting experience is often available in the field of diagnosis.

Motion capture techniques are an emerging technology to measure human motions and analyze his/her intention, i.e. including knowledge. Several commercial motion capture system are available, however they cost around a hundred thousands in US dollars. On the other hand, Human motion learning attracts much attention in the field of vision researchers. For example, Song [7] proposes unsupervised learning method of human motion. Bennewitz [8] also proposes a learning method of human motion for robot teaching. Lee and Mase [9] developed a system of activity and location recognition using wearable sensors, which includes IC accelerometers.

\section{Motion Measurement Algorithm}

This section describes how our method measures human motions. First basic motions are defined as Motion study of Industrial Engineering does. Then IC sensors and signal processing algorithm are described.

\subsection{Motion Study and Therblig}

One of the major objectives of Industrial Engineering (IE) is improving productivity in manufacturing lines. In order to achieve this objective, motion study was established. Motion study is a way of analyzing and improving human motions in a work task. Gilbreth defined 18 basic motions of assemble tasks and named them "Therblig" [10]. As Gilbreth claimed, complex human tasks consist of several basic motions. In maintenance tasks, turning on and off a switch is often considered important events. A service technician reaches his arm as manipulating switches and meters. Therefore we can know important events of maintenance if "Reach" motion can be observed. As a next step we employed IC accelerometers to measure "Reach" motion.

\subsection{Motion Measuring by IC sensors}

We employed IC accelerometers to measure "Reach" motion. This section describes IC accelerometers and its signal processing. Analog Device's ADXL202 is employed as an accelerometer. The ADXL202 are low cost, low power, complete 2-axis accelerometers with a measurement range of $\pm 2 \mathrm{~g}$.

Two ADXL sensors are attached to a right arm as figure 4 shows. 


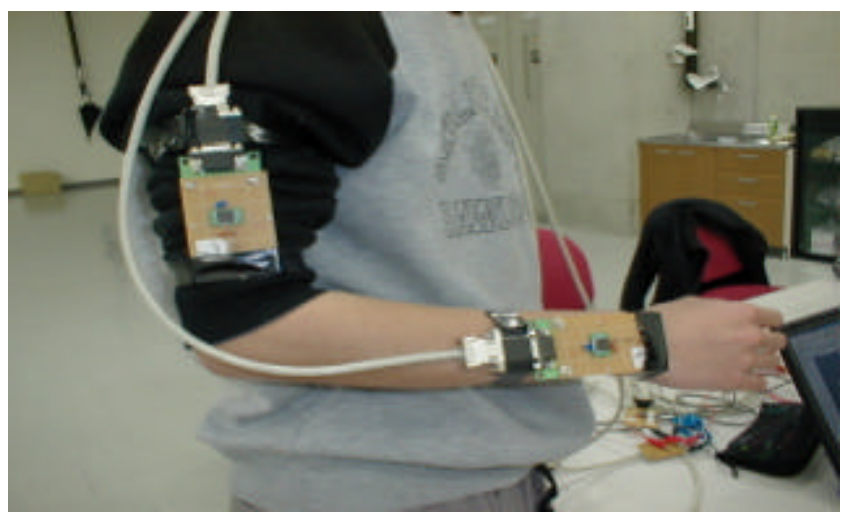

Fig. 4. Accelerometers on Right Arm

Figure 5 demonstrates intuitively How "Reach" motion is detected from the output of wrist ADXL. Right hand are first put aside at a leg. In "Reach" motion, the hand raises and falls. This movement is clearly recorded with ADXL's X and Y axes sensors.

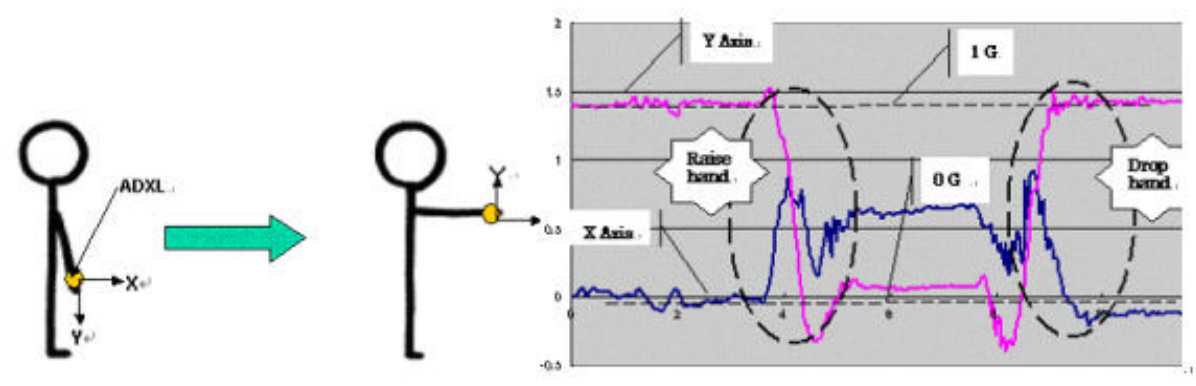

Fig. 5. Wrist Accelerometer's Output

To detect a basic motion from this data, the output signal is processed as below:

Step-1 Raw X, Y acceleration data (50msec Sampling)

$$
x_{1}^{0}, x_{2}^{0}, \cdots x_{N}^{0} ; y_{1}^{0}, y_{2}^{0}, \cdots y_{N}^{0}
$$

where $\mathrm{N}$ denotes the size of sampled data.

$x_{i}^{0}$ : Superscript 0 denotes raw data. Subscript i means i-th data of $\mathrm{X}$-axis.

Step-2 Eliminate noise by averaging

$$
x_{i}^{1}=\frac{x_{i-2}^{0}+x_{i-1}^{0}+x_{i}^{0}+x_{i+1}^{0}+x_{i+2}^{0}}{5}
$$

Step-3 Labeling: Classify each data in to 5 bins, i.e., “-1", “- 0.5 ", “ 0 ", " 0.5 ", and " 1 ". Step-4 Classify: Find the transition of which data becomes -1 to 0 . This transition denotes change of arm direction. Therefore we can conclude "Reach" motion occurs. 


\subsection{Experimental Results}

Motion measurement experiment was conducted with 8 people. The signal-processing algorithm detected all of 56 "Reach" motions of the 8 people while they performed the start-up procedure of an industrial robot system that contains 7 Reach motions.

Therefore we conclude our method is reliable enough to detect a special motion, e.g., "Reach", during a service task.

\section{Conclusion}

After-sales service is one of promising business sectors. Service experience sharing is proven to improve service productivity. However recording service cases costs high and is a bottleneck of knowledge and experience sharing systems. We proposed a framework that records after-service experience and elicits knowledge from the experience. The proposed method employs IC accelerometers to measure human motions. The empirical results prove that the IC sensors and developed algorithm are able to measure and detect the basic motions while a service technician executes his task.

As the next step, we will continue working to develop the inference mechanism of step 3 in figure 2, which infers service task from a series of observed basic motions.

\section{References}

1 E.H. Shortliffe: "Computer Based Medical Consultations, MYCIN", Artificial Intelligence (1976).

2 K.J.Hammond: “CHEF: A Model of Case-based Planning”, Proc. AAAI86, pp267-271 (1986).

3 S.Hori, et.al., "Utilizing Repair Cases of Home Electrical Appliances", IJCAI Practical Use of CBR Workshop, pp.41-52 Nagoya (1997).

4 S.Kobayashi, K.Nakamura, "Knowledge Compilation and Refinement for Fault Diagnosis", IEEE Expert, Vol.6, No.5, pp.39-46, Oct. (1991).

$5 \quad$ B.Kuiper: "Qualitative Simulation" Artificial Intelligence Vol.2 pp.289- 338 (1986).

6 J.R.Quinlan: "Decision Trees and Decision making", IEEE Trans. on Systems, Man and Cybernitics, Vol.20,No.2, pp.339-346 (1990).

7 Song, Goncalves, Perona, : “Unsupervised learning of human motion”, IEEE Transactions on Pattern Analysis and Machine Intelligence, Vol.25, Iss.7, pp.814-827 (2003).

8 Seon-Woo Lee, Mase: “Activity and location recognition using wearable sensors", IEEE Pervasive Computing, Vol.1, Iss.3, pp.24-32 (2002).

9 Bennewitz, Burgard, Thrun: "Using EM to Learn Motion Behaviors of Persons with Mobile Robots", 2002. IEEE/RSJ International Conference on Intelligent Robots and Sy stem, Vol.1, pp.502-507 (2002).

10 Barnes, "Motion and Time Study", John Wiley \& Sons, Inc., (1968). 